\title{
La Fueguía Antártica de Enrique Delachaux ¿Puente o barrera geográfica Austral? ${ }^{1}$
}

\author{
The Antarctic Fueguía by Enrique Delachaux. \\ Austral geographic barrier or bridge? \\ MAURICIO JARA FERNÁNDEZ \\ "Universidad de Playa Ancha, Valparaíso \\ $>$ mjara@upla.cl
}

\begin{abstract}
RESUMEN
Enrique Augusto Samuel Delachaux es uno de los pocos geógrafos sudamericanos que se ocupó -escribió- de la Antártica en los comienzos del siglo XX. Antártida fue una contribución del volumen de homenaje del Instituto Geográfico Argentino en 1904 a los expedicionarios de la corbeta Uruguay que rescataron a los náufragos suecos liderados por Otto Nordenskjold y al héroe antártico argentino José María Sobral. En este estudio Delachaux expone cuatro cuestiones fundamentales, a saber: la importancia de las expediciones a la Antártica, la similitud entre Tierra del Fuego y la península antártica, el encadenamiento orográfico en los mares australes y que la Antártica es un continente y no un mar con tierras polares.
\end{abstract}

PALABRAS CLAVE: Antartandes, Continuidad Geográfica, Fueguía Antártica.

\begin{abstract}
Enrique Augusto Samuel Delachaux is one of the few South American geographers who dealt -he wrote- of Antarctica in the early twentieth century. Antarctica was a contribution of the volume of tribute of the Argentine Geographic Institute in 1904 to the expeditionaries of the corvette Uruguay who rescued the Swedish castaways led by Otto Nordenskjold and the Argentine Antarctic hero José María Sobral. In this study Delachaux exposes four fundamental questions, namely: the importance of expeditions to Antarctica, the similarity between Tierra del Fuego and the Antarctic Peninsula, the

1 Proyecto Conicyt-Fondecyt Regular No 1170314, titulado "El Piloto $2^{\circ}$ Luis Pardo Villalón y la Segunda Carta Patente Británica: La Política Antártica Chilena entre la Pertenencia Histórica y la Incertidumbre Internacional, 1906-1917”.


orographic chaining in the southern seas and that the Antarctic is a continent and not a sea with polar lands.

KEY WORDS: Antartandes, Geographic Continuity, Antarctic Fueguía.

\section{INTRODUCCIÓN}

A fines del siglo XIX el continente Antártico comenzó a despertar un insospechado interés en las academias científicas europeas por ser uno de los pocos espacios del orbe que todavía estaba bajo un manto de misterio y sobre el cual había muchas interrogantes no resueltas. Por esta subsecuente inquietud científica y los propios llamados efectuados por estas academias, rápidamente se organizaron expediciones a los mares y tierras antárticas, incluso, algunas, con apoyos políticos y financieros de sus respectivos gobiernos para llegar a tener una mayor exactitud de sus costas, formas y características físicas y, fundamentalmente, para intentar penetrar hacia el interior del continente y conquistar el Polo Sur (Day, 2013, pp. 98-124).

En este quehacer expedicionario antártico, la explotación de recursos marinos no fue algo excepcional sino más bien regular, puesto que permitía compensar los gastos efectuados. A este respecto, no deja de llamar la atención que la Sociedad de Geografía de Hamburgo declarara en 1894 que "se ha dado lectura al diario de navegación de tres buques balleneros a vapor, la Herta, el Jason y el Castor, que han recorrido las altas latitudes australes durante los dos años 1892 y 1893, por cuenta de una sociedad de armadores de ese puerto”.²

Varias fueron las expediciones europeas que zarparon en dirección a las costas y tierras antárticas al finalizar el siglo XIX y en los albores del siglo XX (Woodward, 1902). A las emprendidas por Carsten Borchgrevinsk, Robert Scott, William Bruce y Erich von Drygalski, las de Adrian de Gerlache en la Bélgica y la de Otto Nordenskjold en el Antarctic fueron las que mayor impacto e interés causaron en la población y autoridades de Punta Arenas (Jara, 2013) y Buenos Aires, respectivamente.

Esta última, tanto por el salvamento humanitario realizado por la corbeta Uruguay a los acompañantes de Nordenskjold y, muy especialmente, por las nuevas informaciones científicas aportadas sobre la Antártica llevaron al geógrafo de origen suizo, nacionalizado argentino, Enrique Augusto Samuel Delachaux ${ }^{3}$, a redactar un sugerente y casi desconocido artículo Antártida (Delachaux, 1904, pp. 143-160), publicado en el Boletín del Instituto Geográfico Argentino (BIGA) en 1904. Ese año el BIGA, celebró y homenajeó el éxito alcanzado por la corbeta Uruguay de la Armada Argentina al rescatar a los expedicionarios suecos del

\footnotetext{
“Esploración en los Mares del Sur”. El Magallanes (Punta Arenas) 3 junio 1894, p. 2.

Nació en Neuchátel, Suiza, el 18 de abril de 1864 y murió en La Plata, Argentina, el 10 de abril de 1908. Estudió en la Escuela de Dibujo y Matemáticas en Yverdon, comuna suiza del cantón de Vaud, capital del distrito de Jura-Nord vaudois. Luego realizó cursos en la Universidad de La Sorborna y en el Museo de Historia Natural de París. Fue colaborador y asesor del perito Francisco Pascasio Moreno en la cuestión patagónica con Chile y en 1899 se entrevistó en Santiago con Diego Barros Arana y Alejandro Bertrand.
} 
Antarctic $^{4}$ (Anguita, 1904, pp. 127-129; Pomar, 1903, pp. 436-438); y al oficial naval y naciente héroe antártico argentino José María Sobral.

Al parecer, el salvamento de los náufragos suecos en noviembre de 1903 y la conferencia dictada en Buenos Aires por el eminente geólogo y líder de aquella expedición polar, Otto Nordenskjold, habrían sido, las dos principales motivaciones que Delachaux tuvo para escribir Antártida y explicar al medio científico argentino de entonces, algunas cuestiones geográficas fundamentales sobre aquella "región de la tierra (que) ha llamado tanto la atención (y) motivado tantas expediciones ... a la zona polar austral” (Delachaux, 1904, p. 143) como las organizadas por Inglaterra, Alemania, Suecia y Escocia en los comienzos del siglo XX. Particular interés despertó en Delachaux la singularidad geográfica que resultaba de la indiscutible continuidad territorial entre los confines americanos y los archipiélagos y tierras antárticas y que él acuñó y denominó 'Fueguía Antártica'.

Dado que para Delachaux (1904, p. 149) la expresión 'Fueguía Antártica' era la más apropiada para denominar "las dos puntas...opuestas, como lo son también los dos continentes": América y Antártica, la hemos utilizado en el título de este trabajo.

$\mathrm{Al}$ analizar Antártida cuatro son las ideas centrales que Delachaux desliza con claridad, a saber: la importancia de las expediciones para la actualización del conocimiento geográfico, la similitud de la península antártica con el extremo meridional americano, la existencia de un encadenamiento insular en el mar austral con forma de arco y el "desvanecimiento" o desactualización científica de quienes han seguido sosteniendo que en la Antártica hay porciones de tierras rodeadas de un gran océano en circunstancias que éste es un continente.

Estas propuestas de Delachaux permiten tener una mejor comprensión sobre la visión geográfica y científica que se tenía en aquellos años sobre la zona austral americana del orbe y la manera en que era posible llegar al Polo Sur. Como se sabe, la llegada del hombre a ese lugar de la Antártica ocurrió siete años después, en diciembre de $1911^{5}$.

Por último, la interpretación científica que Delachaux consignó para abordar la morfología y, especialmente, la dirección que poseían los encadenamientos orográficos insulares en el mar austral -al sur del Cabo de Hornos- permite entender porque esta no logró tener mayor figuración y aceptación científica al momento de presentar la geografía austral-antártica argentina.

\section{DESARROLLO}

Antártida de Delachaux es uno de los pocos estudios sudamericanos sobre esta temática a comienzos del siglo XX (Jara, 2017, pp. 71-74) y en gran parte el análisis geográfico que presenta fue posible gracias a las nuevas informaciones aportadas por los expedicionarios

4 La corbeta Uruguay comandada por Julián Irizar rescató a los náufragos suecos de Otto Nordenskjold. En la expedición de salvamento de la Uruguay participó el teniente de la marina chilena Alberto Chandler Bannen. En: Espedición al Polo Sur (1903). Revista de Marina, 35(205), p. 319; Nordenskjöld y sus compañeros (1903). Revista Chilena de Historia Natural, 7, pp. 334-335.

5 Roald Amundsen de nacionalidad noruega fue quien llegó y conquistó el Polo Sur el 14 de diciembre de 1911. 
suecos de la siniestrada Antarctic en el mar de Weddell en 1903.

Para Delachaux (1904, p. 143), las actividades científicas y exploratorias desplegadas tanto por los gobiernos y los particulares en las tierras australes y antárticas desde los últimos años del siglo XIX eran una inmensa contribución a la humanidad por el "arrojo de los descubridores de nuevos mundos" y porque con ellas en el mapa universal se estaban despejando las zonas desconocidas del planeta ${ }^{6}$.

Aun cuando las zonas templadas y tórridas del planeta seguían a la espera de mejores y más completos estudios, los descubrimientos australes estaban de moda y eran una muy buena oportunidad para avanzar en el conocimiento científico de una región meridional que ya era llamada por todos: el sexto continente, las tierras polares australes, el gran continente austral o el continente blanco ${ }^{7}$.

Las expediciones australes se habían intensificado luego del Primer Año Polar (1882-1883) y "Después de las revelaciones que nos han hecho alcanzar los sacrificios de las expediciones boreales (...) para despejar incógnitas que obstaculizan la solución de problemas trascendentales, ... hoy las expediciones australes quedan en mucho facilitadas, merced a la ocupación permanente ... de las tierras extremas del continente americano. De estas a la tierra de Graham puede decirse que hay un paso" (Seguí, 1885, pp. 21-21). Hay que recordar que desde Punta Arenas en 1897 la expedición de Adrian de Gerlache en la Bélgica logró completar provisiones y el descanso de la tripulación antes de zarpar en dirección a la Antártica (Jara, 2013).

A diferencia de lo que había ocurrido con el Ártico, las grandes expediciones científicas que pretendían acceder al Polo Sur geográfico estaban recién organizándose ${ }^{8}$ debido en parte importante a "la misma configuración de ambos hemisferios: en el boreal están concentradas casi todas las tierras, ocupadas por mayor parte de las naciones civilizadas, mientras en el austral predomina el elemento líquido” (Delachaux, 1904, p. 146), lo cual hacía más difícil el reconocimiento de sus costas y penetrar al interior.

Por 1904 ya había cierta certeza científica que el Ártico era una gran tierra o continente boreal y que se asemejaba a "una depresión marítima helada en aquel eje del mundo" (Delachaux, 1904, p. 145) mientras que en la antípoda, la Antártida, las expediciones científicas todavía no lograban sobrepasar la zona templada y únicamente habían llegado a pocos kilómetros de la zona glacial alcanzada por el Gauss de Drygalsk ${ }^{9}$. Y, al igual como ocurría en los mares y tierras boreales, estas expediciones australes junto a la búsqueda de

6 Delachaux conocía bastante bien las expediciones realizadas a la Antártica y asignaba importantes méritos a las primeras efectuadas por Palmer, Weddell, Bransfield, Biscoe y Larsen. En los últimos años y siendo fundamentalmente científicas, las de Cook, Ross, Wilkes, Dumont D’Urville, Nares, Gerlache, Borchgrevinsk, Scott, Nordenskjold, Bruce y Drygalski.

7 Delauchaux usaba indistintamente estas expresiones para referirse a la Antártica.

8 La expedición del teniente de navío Adrian de Gerlache en la Bélgica en 1897-1899 fue la primera en invernar en la Antártica y la que más atractivo causó en Punta Arenas tanto en la ida como al regreso.

9 La expedición del Gauss, buque tipo bergantín-goleta, estuvo comandada por el geólogo y experimentado explorador del ártico Erich von Drygalski y fue la primera expedición alemana en la Antártica entre 1901 y 1903. Exploró la zona antártica al sur de las islas Kerguelen y descubrió la Tierra del Emperador Guillermo II y el volcán Gaussberg. Se reconoce a Drygalski el haber usado por primera vez el globo aerostático en la Antártica. 
nuevos conocimientos geográficos también realizaban actividades cazadoras de focas o lobos (p. 146) para contribuir al financiamiento de estas y para tratar de asegurar la continuidad de los trabajos de investigación.

En este sentido, los nuevos descubrimientos y conocimientos entregados por los expedicionarios rescatados del Antarctic, habrían sido decisivos para Delachaux al momento de escribir su artículo y en orden a postular la hipótesis que la península antártica poseía una sorprendente similitud orográfica con el extremo meridional americano. Hay que recordar que el propio Otto Nordenskjold en 1897 (pp. 157-168), de visita en Chile y Argentina, había planteado la necesidad de seguir investigando la geografía física de la Tierra del Fuego con los territorios adyacentes australes, conforme a lo que también había postulado en 1893 Francisco Fonck (p. 2) que los volcanes en la costa del Pacífico se extendían hasta el Cabo de Hornos, las islas Shetland del Sur y la Tierra de Graham. Casi diez años después, en 1902, el mismo Fonck (p. 76) nuevamente reiteraba que la cordillera de los Andes de norte a sur proyectaba una continuidad geográfica hacia la región subantártica, la cual se ubicaba al sur del territorio de Magallanes, donde Punta Arenas era "la población más meridional del mundo, centinela avanzado de la civilización en las tierras que avecinan al polo” (Lista, 1881, pp. 57-59) y en dirección al archipiélago de las islas Shetland del Sur.

También, Delachaux, observaba que la similitud existente entre el extremo meridional sudamericano con la península Antártica no se reproducía en ningún otro continente situado en el hemisferio sur y, lo único que era posible advertir a nivel general que Australia y el mismo Brasil con el continente antártico, presentaban una especie de sincronización de ensamble de sus costas como consecuencia de haber estado unidas en algún momento de la historia geológica del planeta $^{10}$.

Las llamadas Tierra de Luis Felipe, Tierra de Palmer, Tierra de Danco, Tierra de Oscar II y Tierra de Graham, hasta 1903, decía Delachaux, habían sido solo exploradas hasta los $66^{\circ}$ latitud sur y aparecían en las cartografías como una península continúa hacia esa latitud, sin embargo, nada aseguraba que, avanzando hacia latitudes mayores, aparecieran "series insulares como sucede en la Tierra del Fuego y regiones vecinas” (Delachaux, 1904, p. 150) y esta se interrumpiera. La incógnita, en consecuencia, era saber si acaso la península antártica tendría continuidad terrestre u orográfica hacia el interior del continente. Esta respuesta científica Delachaux no la conoció pues falleció en 1908 e incluso se logró tener muchos años después que el hombre alcanzó el Polo Sur geográfico ${ }^{11}$.

En todo caso Nordenskjold, en la conferencia que dictó en un famoso teatro de Buenos Aires y ante numerosos asistentes ${ }^{12}$, en diciembre de 1903, dijo que él creía haber “establecido positivamente la unidad de aquella alargada península, que tan asombrosa

10 PANGEA, era un supercontinente que agrupaba a diversas porciones emergidas de la tierra y que existió entre fines de la era paleozoica y comienzos del mesozoico.

11 La llegada de Roald Amundsen al Polo Sur geográfico en diciembre de 1911 no resolvió la interrogante de si la península se interrumpía a mayores latitudes o si era una masa de tierra separada del resto del casco antártico.

12 Nordenskjold hizo su presentación en el teatro Politeama de Buenos Aires y a ella asistió el teniente chileno Alberto Chandler Bannen. 


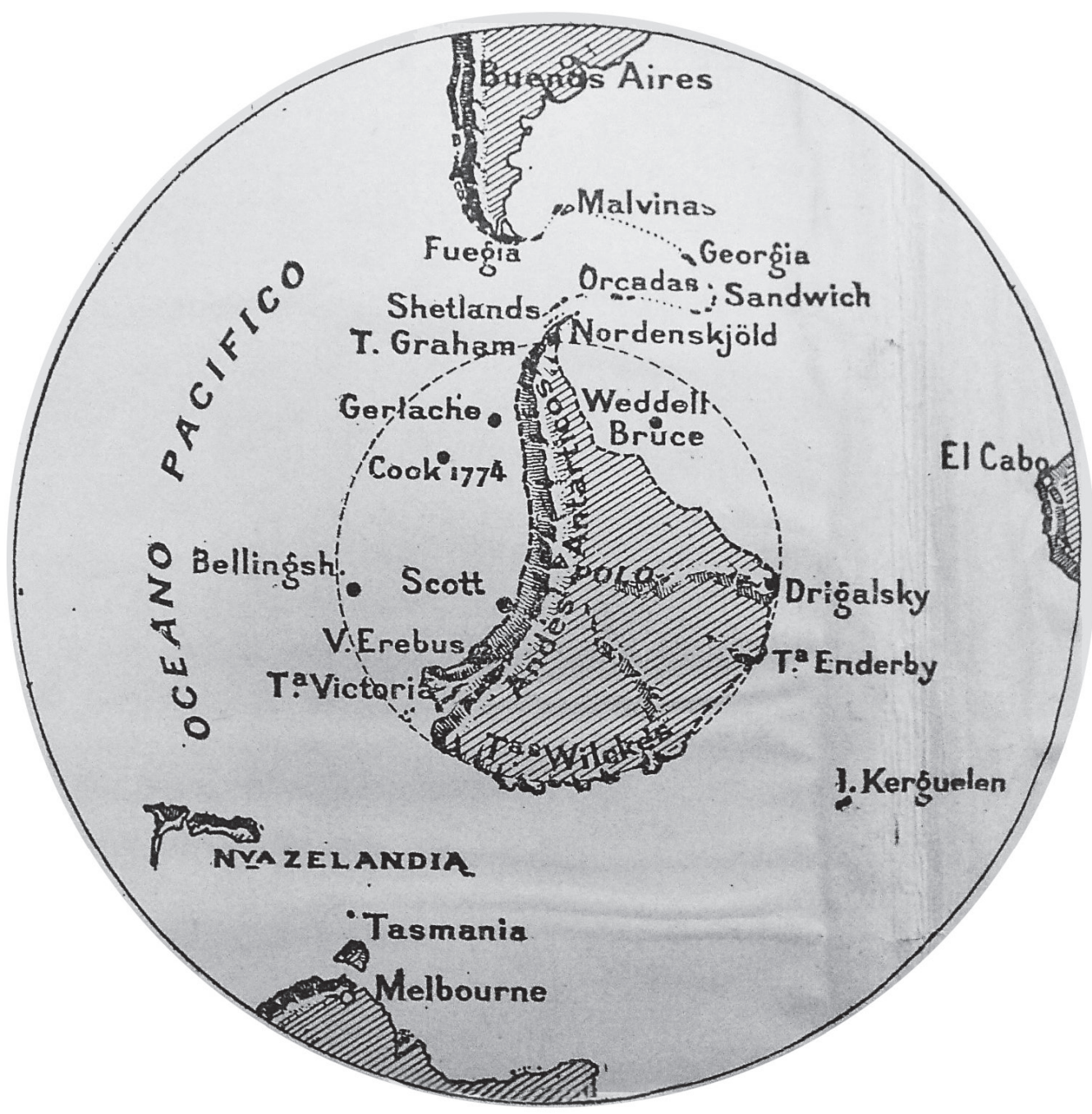

Figura 1: Antártida

Fuente: E. Delachaux (1904). Boletín del Instituto Geográfico Argentino. Tomo XXII, Buenos Aires.

analogía tectónica presenta con la Tierra del Fuego" (Delachaux, 1904, p. 148). (Fig. 1). Era la primera vez que un geólogo y expedicionario antártico llegaba a esa conclusión y afirmaba que en la "Patagonia andina, existen aquí alineamientos insulares paralelos a la orilla y que representan exactamente las precordilleras del continente Sudamericano" (p. 150).

Por la trascendencia científica de la propuesta que Nordenskjold expuso sobre la península Antártica, Delachaux pensaba que, esta debería llevar el nombre del científico que acompañó a Gerlache en el Bélgica y que falleció allá: Tierra de Danco, "pero si nos atenemos a semejanzas físicas, es preciso confesar que ningún nombre le corresponderá mejor que el de Tierra del Fuego Austral o Fueguía Antártica por la semejanza entre aquellas dos extremidades continentales" (p. 149). Sin embargo, en el contexto morfológico de la "lengua terrestre estrechada y con extraordinario adelgazamiento de la Península Graham-Danco-Luis 
Felipe” (p. 150), Delachaux observaba que el encadenamiento orográfico que emergía del mar austral en las cercanías de la propia península era muy especial pues: "El globo ofrece pocos - tal vez ningún otro - ejemplos de una lengua terrestre tan estrechada y es extraño que ni Dumont d' Urville, ni Palmer, ni Bransfield, que llegaron por el Pacífico tan cerca del punto en donde el adelgazamiento es mayor, pudieran comprobar esta circunstancia dándose cuenta de que unos 8 kilómetros más allá encontrábanse las aguas del Atlántico” (p. 150).

Para Delachaux lo relevante de este último aspecto era como Palmer, Bransfield y Dumont d'Urville, quienes habían navegado desde el Pacífico hacia el levante no habían reparado en que aquellas islas alineadas y emergentes en el mar austral y en conexión con la península antártica pudieran separar geográficamente las aguas de los océanos Pacífico y Atlántico. Esta observación de Delachaux que, en apariencia, pudiera ser interpretada como marginal en el conjunto de su estudio ${ }^{13}$, indiscutiblemente, fue una inmensa contribución a la dinámica oceanográfica en esas latitudes y a la geografía física austral y antártica, ya que "los sondajes efectuados entre la Tierra del Fuego, las Shetlands y el archipiélago de las Sandwich australes, establecen de una manera casi concluyente la desviación de aquel gran conjunto orográfico hacia el Este, pasando la cordillera, ora terrestre, ora submarina, por los grupos insulares de Islas de los Estados, Malvinas, Georgias del Sur, Sandwich del Sur, Orcadas del Sur y Shetlands Australes" (Delachaux, 1904, pp. 153-154; Ihl, 1953, pp. 45-51) ${ }^{14}$.

Respecto de la Antártica, Delachaux, advertía con propiedad científica que "encontraremos allí una exacta repetición del sistema orográfico Sudamericano" (p. 150) ${ }^{15}$ y que como continente independiente presentaba una semejanza con América del Sur (p. 151) y debiera ser un acontecimiento geográfico de la mayor importancia al convertirse en "el pivote central, la punta fundamental de las tres unidades sólidas del mundo" (p. 151).

A propósito de esta última reflexión, Delachaux aventuraba una hipótesis - que con el tiempo se confirmará - donde decía: “Las secciones montañosas entrevistadas por Scott en el Sur de la Tierra Victoria, hasta unos 8 grados del polo, y las reconocidas por Nordenskjold en la Tierra de Palmer no son sino partes de una inmensa cordillera que atraviesa todo el Continente Blanco, de punta a punta, como lo hacen los Andes en la América Austral” (p. 152).

Probaban lo dicho "Las observaciones recogidas por las expediciones inglesa y sueca nos autorizan a ... señalar en su lugar la cordillera polar que llamaremos Andes Antárticos o Cordillera Américo-australiana, pues establece verdaderamente la unión entre los dos citados continentes" (p. 153).

13 Llama la atención que en los estudios referidos a la Antártica Argentina pocas veces aparece mencionada y menos aún citada, la Antártida de Enrique Delachaux; habrá sido que esas observaciones de la geografía física austral y antártica no han acomodado suficientemente a las interpretaciones geográficas o pretensiones territoriales trasandinas en esa zona meridional y antártica.

14 Las islas a las que aludía Delachaux son las mismas que agrupadas recibieron más tarde el nombre de Arco de Scotia y que ha sido utilizado como un hito o accidente geográfico austral para dividir los océanos Pacífico y Atlántico en esas latitudes.

15 Delachaux en diciembre de 1901 planteó la existencia de una cordillera en la Antártica cuando todavía nadie se atrevía a formularla en base a la teoría del tetraedro. 
Las bajas temperaturas y los fríos intensos en la estación de invierno en la Fueguía Americana y Fueguía Antártica, o sea, en el mismo Continente Blanco, eran para Delachaux similares a las que ya se conocían de Alaska e inmediaciones, en torno a los $52^{\circ}$ bajo cero (p. 157) ${ }^{16}$. Esta era la principal característica meteorológica del sexto continente del planeta.

La Antártica, en definitiva, era en 1904 vista como un vasto continente, poseedor de abundante plancton en sus mares adyacentes y con la "misma configuración que la América del Sud, atravesada como ella, y a la vista de la costa del Pacífico, por una inmensa y elevadísima cordillera que no es sino la continuación de la andina y de la australiana, siendo muy posible que el mismo polo ocupe una de las cumbres más elevadas y más centrales de dicha cordillera" (Delachaux, 1904, p. 159) y donde probablemente algún día no muy lejano iba a ser posible encontrar el pivote central del mundo, el Polo Sur (p. 160).

\section{CONCLUSIONES}

El estudio Antártida de Delachaux es parte del homenaje dado por el Instituto Geográfico Argentino al rescate de la cañonera Uruguay a los náufragos suecos del Antarctic en noviembre de 1903.

Delachaux a lo largo del estudio Antártida demuestra ser un geógrafo profesional y muy actualizado de las actividades y el quehacer investigativo en la Antártica.

El concepto de Fueguía Antártica acuñado por el propio Delachaux representa una manera de confirmar la existencia de una continuidad geográfica entre Tierra del Fuego, los archipiélagos y la península antártica, pudiendo ser perfectamente aceptable la idea científica de un puente entre ambos continentes.

El encadenamiento orográfico emergente en el mar austral que con el tiempo recibirá el nombre de barrera o 'Arco de Scotia' y que permite separar geográficamente los océanos Pacífico y Atlántico no fue, por cierto, una propuesta o interpretación geográfica que pudiera tener una fácil aceptación por los intelectuales o geógrafos argentinos en 1904, puesto que trasladaba las aguas del Pacífico más allá del meridiano que Argentina pretendía en esa zona austral y Antártica. Un tema ampliamente debatido por las sociedades de geografía y círculos políticos en Chile y Argentina durante el curso del siglo XX por la relevancia y trascendencia que tuvo para validar el principio de Chile en el Pacífico y Argentina en el Atlántico.

La Antártica como continente único - el sexto - estaba atravesado por la cordillera polar antártica o Antartandes y tenía una extraordinaria similitud geográfica con América del Sur.

Al rescatar del pasado - o poner en valor - a un geógrafo suizo-argentino que redactó uno de los primeros trabajos sudamericanos sobre la Antártica en 1904 y donde hay cuatro propuestas que aún mantienen vigencia científica para la disciplina geográfica, resulta

16 El doctor Supan afirmaba que el Polo absoluto del frío debía encontrarse en las tierras australes. 
necesario advertir que Antártida es anterior a la llegada del hombre al Polo Sur geográfico, lo cual ocurrió en diciembre de 1911. He ahí el mayor mérito que adquiere su visión geográfica sobre la zona austral americana y antártica.

\section{REFERENCIAS}

Bernabé, A. (1904). La Expedición de la Uruguay. Revista Chilena de Historia Natural, 8, 127129.

Bertrand, A. (1884). Nuevo Mapa de Chile, Trabajado Aquí. Anales de la Universidad de Chile, 66, 656-660.

Caballero, L. (1903). El Primer Viaje de Reconocimiento al Sur. Revista de Marina, 35(205), 249-252.

Charcot, J. (1910). Le Pourquoi-Pas dans l'Antarctique.suivi des rapports scientifiques des membres de letat-major preface de M. Paul Doumer. Paris: Ed. Flamarion.

Day, D. (2013). Antarctica A Biography. Oxford University Press. New York.

Delachaux, E. A. S. (1904). Antártida. Boletín del Instituto Geográfico Argentino. Buenos Aires, 22, 143-160.

Delfín, F. (1903). Carábidos Nuevos Chilenos Descubiertos por la Expedición Antártica Belga. Revista Chilena de Historia Natural, 7, 229.

Espedición al Polo Sur. (1903). Revista de Marina Tomo, 35(205), 319.

Esploración en los Mares del Sur. (1894, junio 3). El Magallanes, p. 2.

Fonck, F. (1893). Introducción a la Orografía y Jeología de la Rejión Austral de Sud-América. Valparaíso: Carlos Niemeyer, Editor.

Fonck, F. (1902). Examen Crítico de la Obra del señor Perito Arjentino Francisco P. Moreno. Valparaíso: Carlos Niemeyer Editor.

Gajardo, I. (1903). Un Socorro Oportuno a los Expedicionarios del Antarctic. Revista de Marina, 204, 703-708.

Gajardo, I. (1905). Por los Mares Australes. Reminiscencias de la Primera Campaña del Antarctic a las Tierras del Rey Oscar II. Revista de Marina, 38(227), 640-649.

Ihl Clericus, P. (1953). Delimitación Natural entre el Océano Pacífico y el Atlántico en Resguardo de nuestra Soberanía sobre la Antártica y Navarino. Revista Geográfica de Chile Terra Australis, Santiago, 9, 45-51.

Jara, M. (2017). La Antártida Americana de Luis Riso Patrón a Comienzos del Siglo XX. Anales del Museo de Historia Natural de Valparaíso, 30, 71-74.

Jara, M. (2013). Gerlache's Belgique in the Social Imaginary of Punta Arenas, 1897-1899. Revista Estudios Hemisféricos y Polares. 4(3), 200-210.

L. C., La Reciente Expedición Inglesa al Polo Sur. Revista Zig-Zag, Año V, n² 217.

Lista, R. (1881). Punta Arenas y sus Alrededores. Boletín del Instituto Geográfico Argentino. Buenos Aires, 2, 57-59. 
Nordenskjöld, O. (1897). Algunos Datos sobre la Parte Austral del Continente Sudamericano según Estudios hechos por la Comisión Científica Sueca. Actas de la Sociedad Científica de Chile, 7, 157-168.

Nordenskjöld y sus compañeros. (1903). Revista Chilena de Historia Natural, 7, 334-335.

Nordenskjöld, O. (1905). Viaje al Polo Sur. Tomo II, Barcelona: Edit. Maucci.

Pomar, L. (1903). La Expedición Argentina al Polo Sur. Revista de Marina, 35(205), 436-438.

Porter, C. (1900). Los Musgos Colectados por la Expedición Antártica Belga en el Estrecho de Magallanes y Tierra del Fuego. Revista Chilena de Historia Natural, 4, 102-106.

Porter, C. (1903). Dípteros Nuevos Chilenos Descubiertos por la Expedición Antártica Belga. Revista Chilena de Historia Natural, 7, 218-220.

Riccardi, A. C. (1995). Homenaje a Enrique A. S. Delachaux. Revista Museo La Plata, 41-45.

Seguí, F. (1885). Noticias de Exploraciones y Expediciones. Boletín Instituto Geográfico Argentino, VI, 20-21.

Woodward, W. H. (1902). A Short History of the Expansion of the British Empire, 1500-1902. London: Cambridge at the University Press. 